\title{
Estimación de energía neta de lactancia a partir de la producción de gas in vitro inoculada con licor ruminal
}

\author{
Jorge Sobalvarro-Mena ${ }^{1}$, Jorge Elizondo-Salazar² $\bowtie$, Augusto Rojas-Bourillón ${ }^{3}$ \\ 1 Universidad Nacional Agraria, Nicaragua. Email: jlsmjss@gmail.com.ni \\ 2 Estación Experimental Alfredo Volio Mata, Universidad de Costa Rica. Email: jorge.elizondosalazar@ucr.ac.cr \\ 3 Centro de Investigaciones en Nutrición Animal. Universidad de Costa Rica. Email: augusto.rojas@ucr.ac.cr
}

Con el objetivo de determinar el potencial de cinco ecuaciones basadas en la producción de gas in vitro con licor ruminal a 24 horas, se estimó el contenido de energía neta de lactancia (ENl) y se comparó con la estimación mediante el método de referencia del National Research Council (NRC), se realizó un experimento al evaluar cinco alimentos para vacas lecheras. La producción de gas in vitro se estimó por el método de Menke y Steingass al utilizar un equipo automatizado, fermentar muestras de $0.5 \mathrm{~g}$ de materia seca en duplicado con dos réplicas, y química húmeda para estimar la ENl por las ecuaciones del NRC. La producción de gas mostró diferencias significativas entre alimentos $(\mathrm{P}<0.0001)$. La curva de producción de gas acumulado describió las diferencias del valor de ENl en los alimentos evaluados. El alimento balanceado mostró los contenidos más altos de $\mathrm{ENl}$, mientras que el pasto estrella presentó el valor más bajo $(\mathrm{P}<0.0001)$. Las ecuaciones evaluadas mostraron diferente precisión, según el tipo de alimento, la ecuación A de Seker fue más precisa globalmente, con el más alto valor del índice de correlación de concordancia de Lin $\left(\mathrm{r}^{2}=0,92\right)$. Las ecuaciones evaluadas mostraron diferente precisión en la predicción de ENl en forrajes frescos de pasto estrella africana, pasto rye grass y morera, con errores menores a $0.62 \%$ al estimarse con la ecuación B de Seker y ensilado de maíz la ecuación A de Menke y Steingass en alimento balanceado con error de $-1.45 \%$, respecto al valor estimado por el método NRC. La estimación de ENl en alimentos para vacas lecheras a partir de la producción de gas in vitro que selecciona la ecuación acorde al alimento a evaluar, es un método alternativo al método de referencia NRC, por su precisión y ventajas de costo y tiempo.

$\square$ Autor para correspondencia Jorge Elizondo-Salazar: jorge.elizondosalazar@ucr.ac.cr 